\title{
Correction to: miR-431 inhibits adipogenic differentiation of human bone marrow- derived mesenchymal stem cells via targeting insulin receptor substance 2
}

Yanling Wang ${ }^{1,2+}$, Lei Yang ${ }^{3+}$, Xiaofeng Liu ${ }^{1,2}$, Tao Hong ${ }^{1,2}$, Tao Wang ${ }^{3}$, Aiwu Dong ${ }^{1,2}$, Jiangxiong Li ${ }^{1,2}$, Xiaoyuan $\mathrm{Xu}^{3^{*}}$ and Lingling $\mathrm{CaO}^{1,2^{*}}$

Correction to: Stem Cell Res Ther (2018) 9:231 https://doi.org/10.1186/s13287-018-0980-4

The original article [1] contains an error in spelling of author, Yanling Wang's name. The correct version can instead be viewed in this Correction article.

\section{Author details}

'Department of Endocrinology, The First Hospital of Jiujiang City, Jiujiang 332000, China. ${ }^{2}$ Jiujiang Affiliated Hospital of Nanchang University, Jiujiang 332000, China. ${ }^{3}$ Key Laboratory of System Bio-medicine of Jiangxi Province, Jiujiang University, Jiujiang 332000, China.

Received: 12 February 2019 Revised: 12 February 2019

Accepted: 12 February 2019 Published online: 21 February 2019

\section{Reference}

1. Wang $Y$, et al. miR-431 inhibits adipogenic differentiation of human bone

marrow-derived mesenchymal stem cells via targeting insulin receptor substance

2. Stem Cell Res Ther. 2018;9:231 https://doi.org/10.1186/s13287-018-0980-4.

\footnotetext{
*Correspondence: xiaoyuan.china@qq.com; 1309316573@qq.com

${ }^{\dagger}$ Yanling Wang and Lei Yang contributed equally to this work.

${ }^{3}$ Key Laboratory of System Bio-medicine of Jiangxi Province, Jiujiang

University, Jiujiang 332000, China

'Department of Endocrinology, The First Hospital of Jiujiang City, Jiujiang

332000, China
} 